\title{
Identification of Steel Pipe Defect Based on Virtual Instrument
}

\author{
Yin Ming, Ye Xiaohui, Wang Hongxia and Pan Hongbing \\ Department of Electronic Engineering, Naval University of Engineering, China, \\ 569121080@qq.com
}

\begin{abstract}
Aiming at the problem of traditional nondestructive testing steel pipe defect, Ultrasonic testing system based on Virtual Instrument was designed. The hardware platform is the signal source which is based on micro-controller, and the HS4 data acquisition card by USB bus. The software platform adopts the LabVIEW to program by using the multithread to acquire all kinds of signal by real-time. The nondestructive feature was found by using the filter, STFT analysis and joint time domain analysis. The designed system has been used to the pipe defect inspection of some company, which is stable and can achieve an anticipant effect.
\end{abstract}

Keywords: steel defect, nondestructive testing; Hilbert Transform; Virtual Instrument (VI)

\section{Introduction}

The process in forging, welding, use of Metallic Materials, will form a variety of internal defects, the existence and the expansion of which will lead to shortened life of equipment, poor security and reliability. The method of conventional ultrasonic flaw detection in the detection, due to various reasons, classification is difficult and accuracy and precision are affected by man-made factors. The paper presents a modern detection method, which is based on the principle of modern detection techniques. The system is developed by the graphical programming language - - LabVIEW by National Instruments (NI) company, and realized by calling the DLL. This method has more superiority in the test and measurement, data acquisition, digital signal analysis and other areas.

Now non-destructive testing system at home and abroad will focus on how to improve the real-time and accuracy of data processing and exchange. Ultrasonic Wave Testing System based on virtual instrument is a system that produces electromagnetic guided wave which generated wave echo signals from the object. The echo signal can be in realtime acquisition, processing and transmission. While a large number of instrument driver in LabVIEW is provided, but still can't meet the needs of users. In the paper, the method is apparatus using LabVIEW call DLL technology to achieve data acquisition and processing programs to improve the real-time performance, reliability and efficiency in the non-destructive testing.

\section{System Composition and Principle}

Wherever Times is specified, Times Roman or Times New Roman may be used. If neither is available on your word processor, please use the font closest in appearance to Times. Avoid using bit-mapped fonts if possible. True-Type 1 or Open Type fonts are preferred. Please embed symbol fonts, as well, for math, etc. 


\subsection{System Composition}

Non-destructive testing instrument is composed of hardware system and software system. The hardware system is composed of excitation pulse source, sensors, signal conditioning modules, and HS4 DAQ card. The software mainly is the control and analysis software, which is the core of system. That is the Virtual instrument technology significantly different from the old instrument. It is showed in the figure.

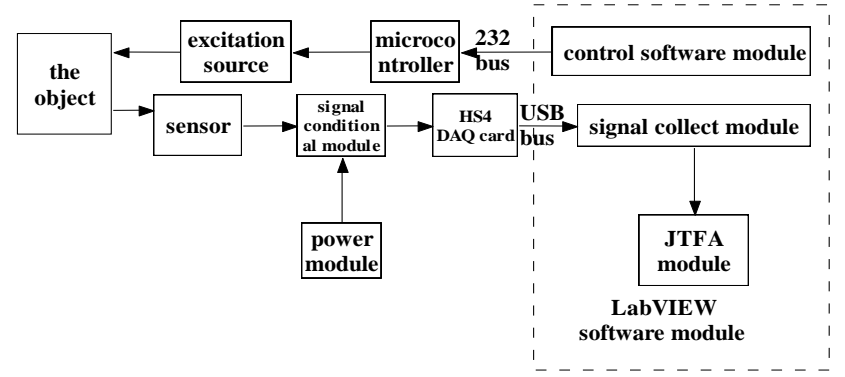

Figure 1. Composition of Non-destructive Testing Instrument

\subsection{System Principle}

The following is the work process of Non-destructive testing instrument.

Firstly, software modules on PC send a command word, which is to the microcontroller through the RS-232 bus. It controls the excitation source to produce electromagnetic wave pulse signal.

Secondly, in the loop of received the echo signal is received by sensor. After the corresponding signal conditioning, the signal is sent to the HS4 DAQ card.

Finally, data through the USB bus is sent into the software module on PC, is acquisition, extracted and processed.

So the key of system is the connect part of hardware system and software system. The paper introduces the method to use testing instrument correctly through LabVIEW program to call DLL, and real-time data acquisition. The following will introduce the HS4 card.

\subsection{Data Acquisition Card- - HS4}

TiePie engineering introduces the Handy-scope model HS4, a portable and compact MOST (Multi-meter, Oscilloscope, Spectrum analyzer and Transient recorder). As four channel measuring device with a $50 \mathrm{MS} / \mathrm{s}$ sample rate, a 12-16 resolution and $128 \mathrm{~K}$ Samples memory per channel, the Handy-scope HS4 is the first four channel MOST in the world which can be connected to a PC (USB2.0 and USB 1.1 compatible), without external power supply. Because of the very extensive and accessible windows based software, the user is offered many measuring possibilities. With good reason you can say the Handy-scope HS4 is really "Plug in and Measure". and it has the driver program for Windows - HS4.dll.

The Key function in the HS4.DLL is followed:

(1) InitInstrument functions: Initialize the hardware of the instrument. Set default measurement settings, allocate memory and obtain the calibration constants etc.

(2) ADC_Start functions: This routine writes any new instrument setting information to the hardware and then starts the measurement. If the hardware is already measuring, this measurement is aborted. Previous measured data is lost.

(3) ADC_Ready functions: This routine checks if the measurement is ready or not.

(4) ADC_GetDataVolt funcitons: This routine transfers the measured data from the acquisition memory in the hardware via the dll into the memory in the application. The 
measured data is returned in volt $\mathrm{dCh} 1$ and $\mathrm{dCh} 2$ are arrays. The caller must ensure that there is enough space in the arrays to contain the data.

\section{Software Design Based on Virtual Instrument}

The software system mainly is achieved by LabVIEW software from NI Company. LabVIEW is the short of Laboratory Virtual Instrument Engineering Workbench. It is a combination of simple-to-use graphical development environment and the $\mathrm{G}$ language of powerful and flexible programming, Provides an intuitive workbench. The user can quickly and conveniently build high applied program on this workbench by defined and linked the icons representing a variety of functional modules.

According to functional requirements, the control and analysis software is mainly divided into incentive pulse generation, echo signal acquisition, defect analysis and data storage characteristics parts. System flow is showed in Figure.

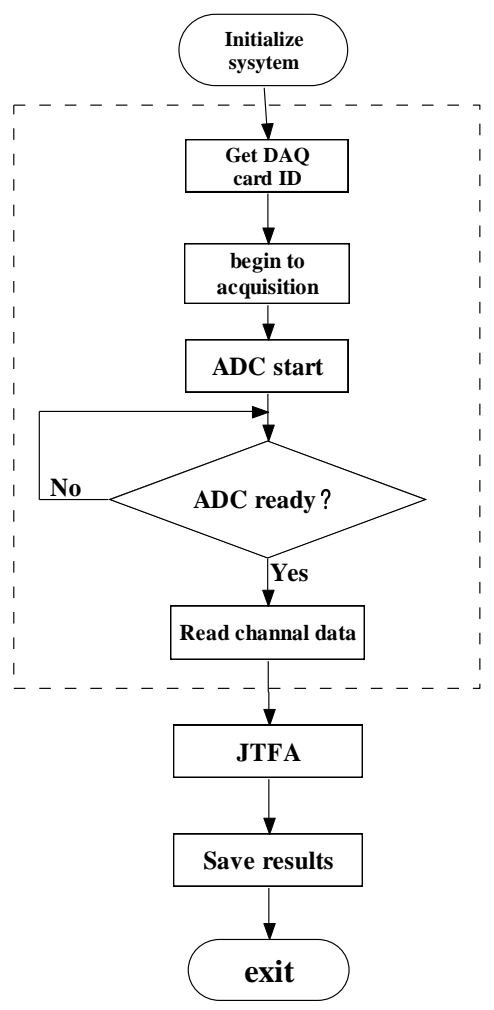

Figure 2. Software System Flow Diagram

\subsection{The Pulse Generation Unit}

In this system the pulse generation function could be set according to the actual applied circumstance by the user. The user keys in each parameter into the dialog box on the operation interface of the control and analysis software, such as, the pulse generation frequency, number of the pulse etc. Then when the user strike the "generate" button and run the program, the system send the parameters that the user has set to pulse generation circuit in the box. That order is sent to the box through serial interface from computer. After the pulse generation circuit received the order the circuit makes sensor create a pulse guided wave in the steel pipe.

The system carries out communication through the serial interface by VISA. First initialize the serial interface that has been appointed by user with the function "VISA configure serial", on the operation interface of the software user could choose the device that the system has found. Then the program cleans the I/O buffer that has been 
appointed, and connects each parameter into a string that the pulse generation circuit could identify, and then sends it to functions "VISA write". The function "VISA write" sends the string to pulse generation circuit. When the parameters have been sent out, the system closes VISA, releases the resources.

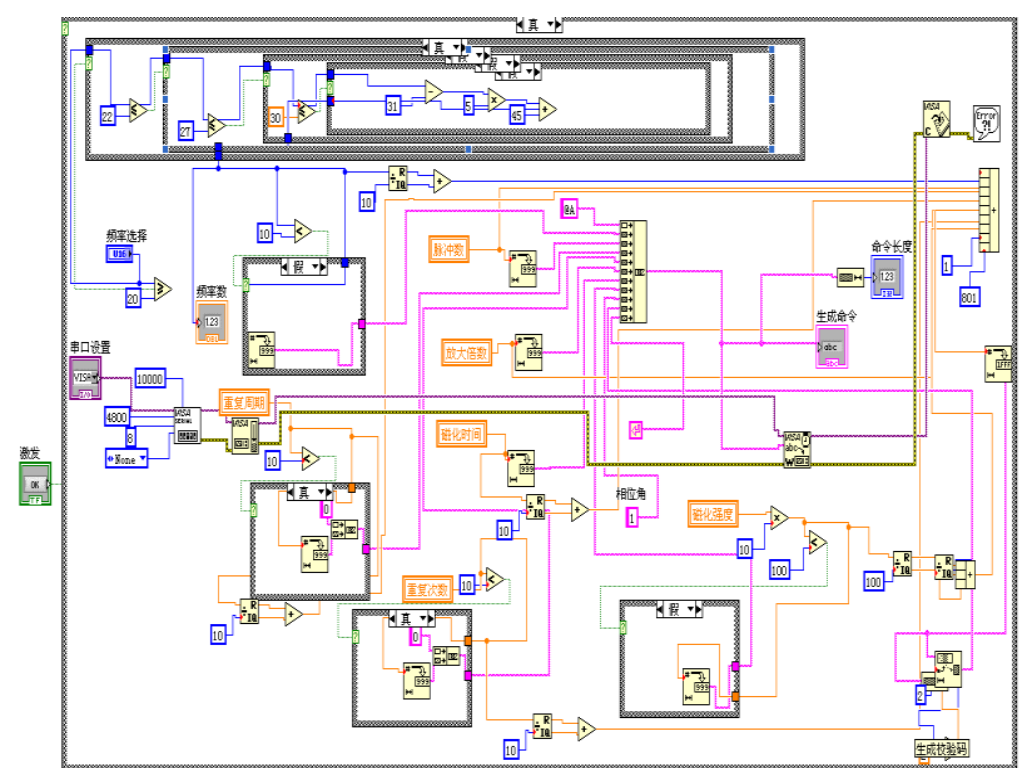

Figure 3. Flow Chart of LabVIEW Software Module

\subsection{Echo Signal Collect}

For example call the HS4.dll, Describes how to implement in LabVIEW real-time collection of information on the ultrasonic wave. Firstly, call InitInstrument function to initialize the hardware of the instrument, get the address and ID. Secondly, set the parameters of sample channel number, sample rate, trigger level and hysteresis-level. Thirdly,call ADC_start functions, to start ADC convert, to trigger the ADC_Ready function by the level of signal acquisition. While triggering, then call data which will be processed and analysis. And store the results. The diagram of data acquisition is show in figure.

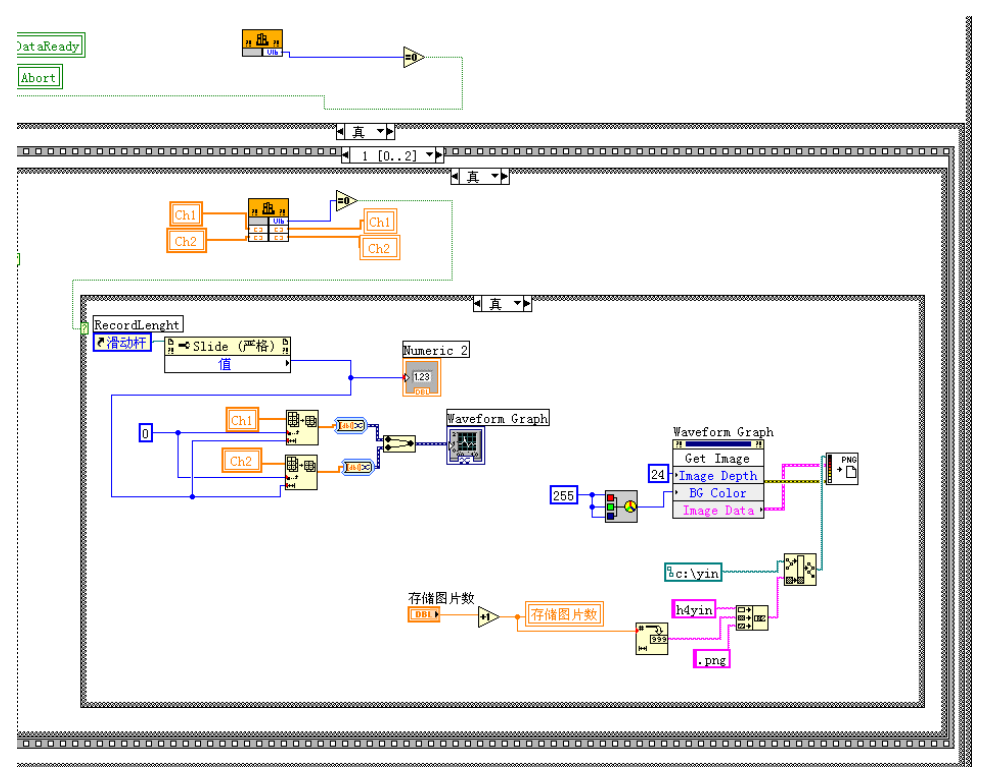

Figure 4. Data Acquisition Block Diagram 


\subsection{Data Store}

Data stored technology: the program store all data thought two kinds of storage forms - - pictures and data. Key technology in the design of the software is used on the following.

(1) Continuous Data Acquisition: in order to realize Continuous Data Acquisition, it must make the system in loop status, then use the while loop. The trigger of data acquisition is trigger level and hysteresis level. While signal arrives the trigger level. System will collect the data.

(2) Using Finite-status machine module: it will be in different status on different command, which is realized by while loop and case structure.

(3) Muti-thread technology: According to requirements of test tasks, it consists of four threads, User interface, signal acquisition, data analysis and processing and data storage. Make full use of the ability of parallel processing of data on LabVIEW.

\section{The Algorithm and Application of Defect Identification}

\subsection{Defect Character Analysis Unit}

At the distance of $2 \mathrm{~m}$ from the end of an steel pipe, we imitate to drill a hole, and detect the defect to it. The echo signal is showed on Figure 5. We can see that there are two defects. Defect signals have been completely submerged into the noise signal. And this, we must remove the noise signal, extracting the useful signal. In this paper, a Butterworth band-pass filter method is used of, bandwidth is $10 \mathrm{KHz}$. Signal filtered is showed in figure.

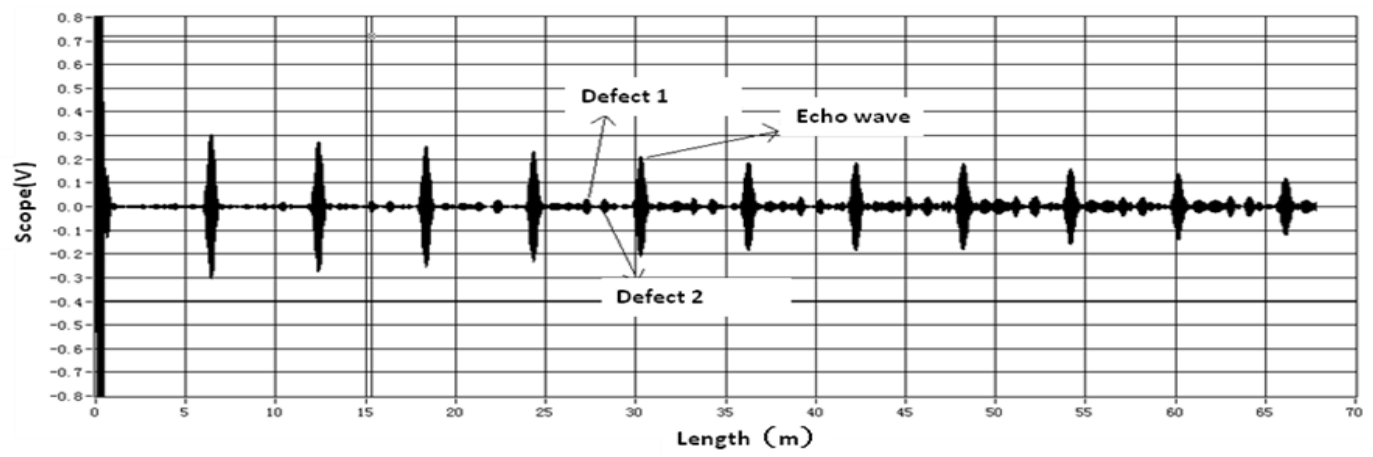

Figure 5. Echo Signal

\subsection{STFT Algorithm}

The signal of ultrasonic echo wave is a non-stationary signal in time domain. It may not fully present its characteristics in time or frequency domain. STFT is by far the one of the most commonly used methods. And it is the one of the most application method in the time domain. It is an extent of FFT transform. STFT is using time window handle to the time signal, then sliding the time window, and making the FFT transform to the little time slice to get the signal spectrum with the times. STFT may solve the problem of frequency domain characteristics within the scope of local time. It is a good choice to analyze the ultrasonic echo signal. 


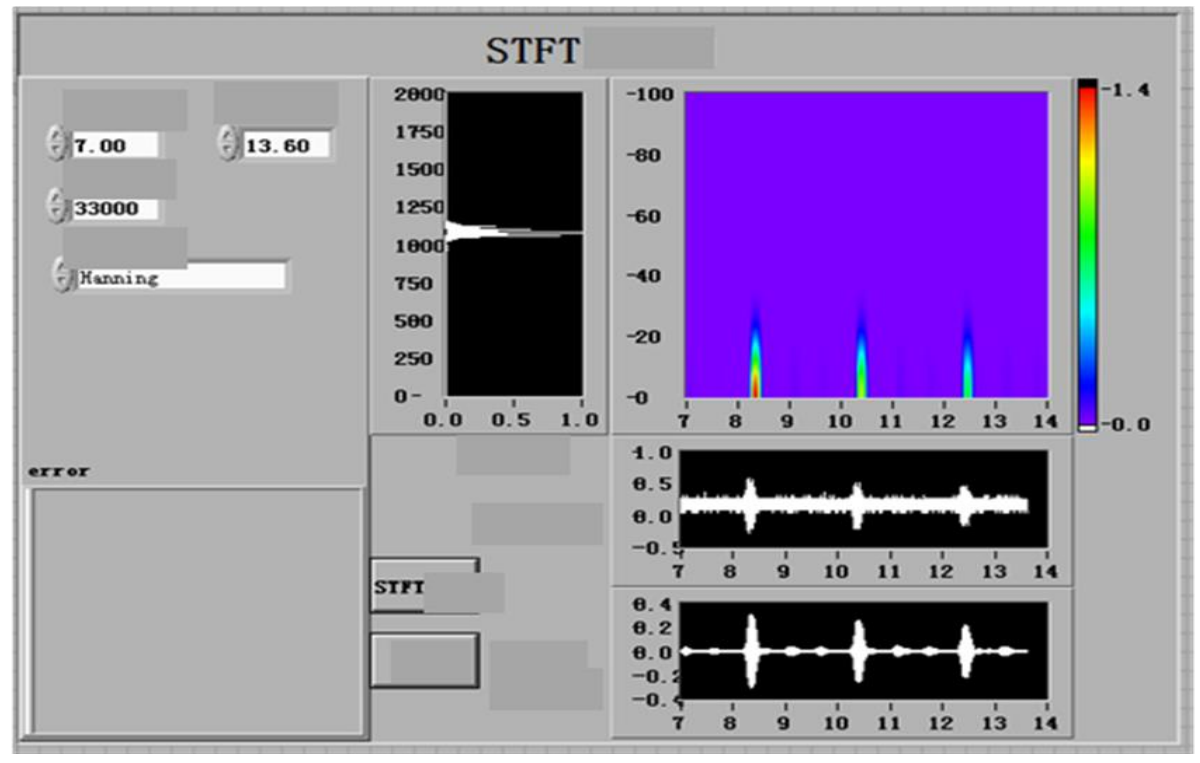

Figure 6. STFT Time-frequency Figure

STFT figure can describe the distribution of energy signal in the time and frequency time-frequency domain. It provides us with a good functional module in LabVIEW software: STFT spectrogram.vi, which function is making use of the principle of shorttime Fourier transform to calculate the energy distribution of the signal in time frequency domain. With this method we can time-domain analysis of joint a number of specific signals, after joint time-frequency analysis of the filtered. Then STFT spectrogram is using in LabVIEW, and using the example of reference related STFT. By writing the program of STFT in time - frequency domain, which analyze the ultrasonic echo signal. Front panel of STFT to analyze signal is showed on the Figure 6. The distribution of the signal energy is showed on the Figure 6.

It adopts frame structure in the program. Firstly, it reads the spreadsheet data and put them into the preset array. It connects with the array and the input of STFT timefrequency figure in the next frame. The output of STFT time-frequency figure is an array of two dimensional. Finally the output terminal connects to the display control.

\subsection{Fast Hilbert Transform}

Hilbert Transform is a kind of transformation that is commonly used in signal processing method. It may be make two-dimensional real signal into a three-dimensional plural and signal processing. It is very helpful to analysis the complex signal, such as modulation of the sine wave. is used to extract the envelope of the ultrasonic signal in the procedures. And take the natural logarithm of the envelope. Then by the Fast Hilbert Transforming the acquisition data make the plural from rectangular coordinate system to the polar coordinate. Take the natural logarithm of radian in the polar coordinate. Procedures to create mainly used the Fast Hilbert Transform function in LabVIEW software.

\subsection{Defect Location}

In order to accurately position the defect, point by point analysis is carried out on the acquisition data. And extract possible defect, find that point corresponds to the actual location. It is showed on the Figure 7 that is the data between any echo on both ends. 


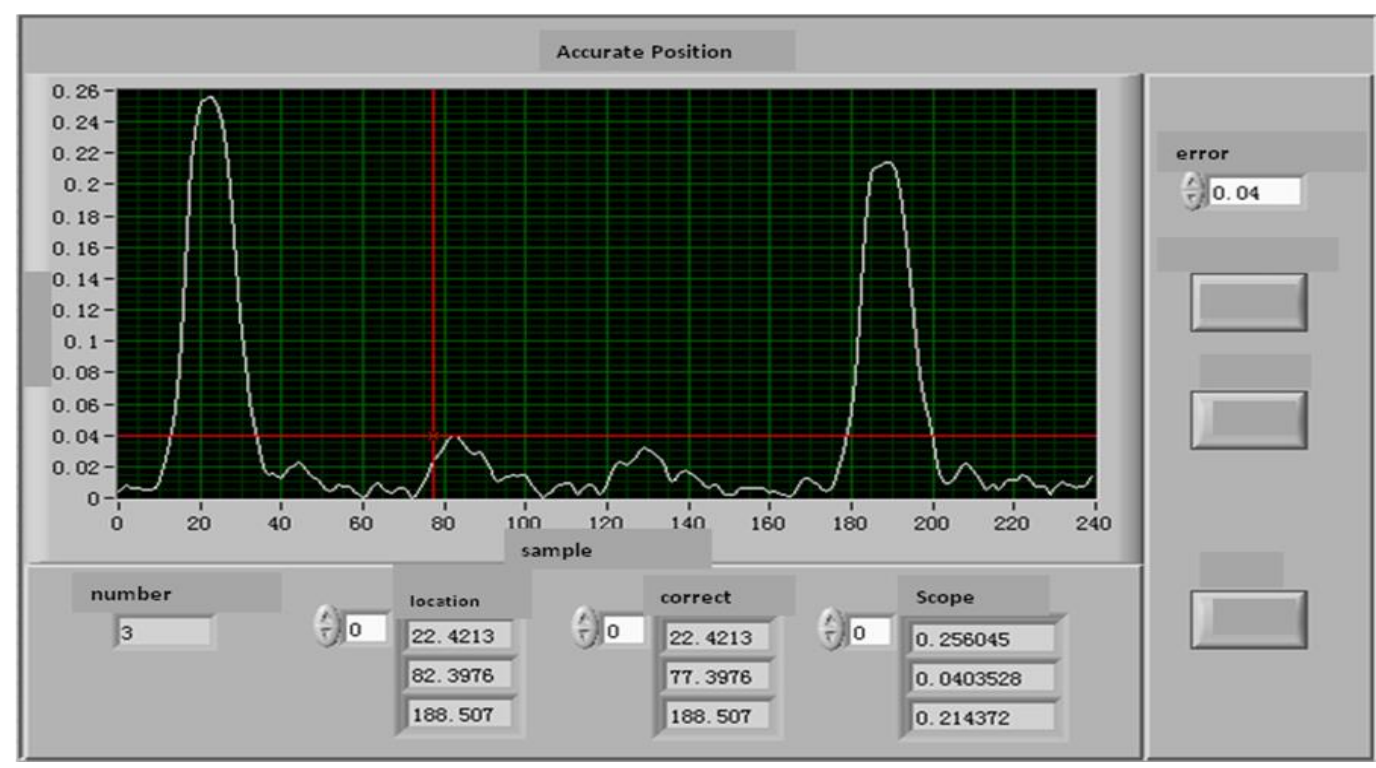

Figure 7. Accurate Positioning

The threshold is set to $0.04 \mathrm{~V}$. After wave detection function, the software may find 3 peaks. The 3 peaks point is showed on the figure 7, which is the crest of two ends and defect echo waveform. We may find the relative position on the horizontal axis about the peaks point. The amplitude of all peaks point may find from the according to the control. The amplitude values of two ends is obviously greater than that of the defect.

Because the defect echo wave location is the actual positing, what is adequate. It needs to correct the relative position of the defect. Correction of specific methods is to bring forward the relative position of defect, which is left a long distance on the oscillogram. The distance is the half of the defect peak. So it is to get the position after correction of defects. For the length of $5.98 \mathrm{~m}$ steel pipe, the location of calculated is the $83 \mathrm{k}$ of excitation frequency. It may calculate the location on the steel pipe in the following.

$$
5.98 m \times \frac{101.16-43.9011}{211.811-43.9011}=2.039 m
$$

For the length of $5.98 \mathrm{~m}$ steel pipe, analysis the acquisition signal for the $77 \mathrm{k}$ of excitation frequency in the following.

$$
5.98 m \times \frac{77.3976-22.4213}{188.507-77.3976}=1.98 m
$$

It used the several times with the average method. Then the error is in the following.

$$
(1.98-2 / 2 \times 100 \%+2.039-2 / 2 \times 100 \%) \times \frac{1}{2}=0.48 \%
$$

So, by multiple measurements for defect location as the actual location, the average accuracy is higher. There are two measurements of steel pipe made and to calculate the average .Position of defect is calculated as $2.0095 \mathrm{~m}$ with the distance of sensor. The error is not more than $1 \mathrm{~cm}$ to the actual position location. The credibility of the positioning is higher.

\subsection{Identify Defect}

We find all kinds of defects on the application of ultrasonic defect detection. There is difference of up edge rate and down edge rate of envelope wave, which may use to identify the character of defect. When the rate of up edge and down edge both is high for the types of crack defect, waveform is steep. However, when the rate of up edge and down edge both is low for the types of loose impurity, waveform is flat. 
In LabVIEW environment the data playback program is implemented to extract the envelope of echo signal. There by using the chart shows the cursor function of control which can calculate up edge rate and down edge rate of the defect envelope. Between two cursors on the vertical axis is the up edge is showed on the figure 8. Usually we consider the $20 \%$ of defect peak as the starting point of up edge and down edge.

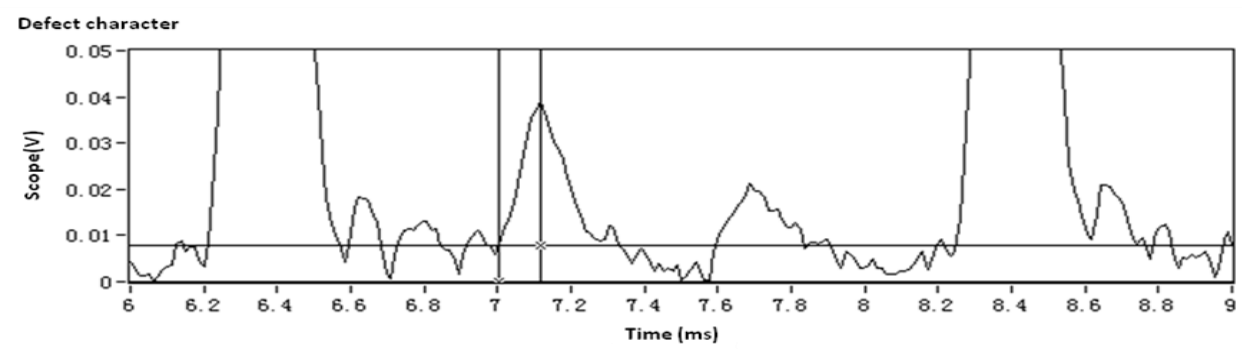

Figure 8. The Up Edge

Form the figure it may calculate the up edge rate a1 and down edge rate a2 of the defect in the measured data respectively.

$$
\begin{aligned}
& a 1=\frac{0.04-0.008(\mathrm{~V})}{0.113(\mathrm{~ms})}=0.283(\mathrm{v} / \mathrm{ms}) \\
& a 2=\frac{0.04-0.008(\mathrm{~V})}{0.213(\mathrm{~ms})}=0.15(\mathrm{~V} / \mathrm{ms})
\end{aligned}
$$

Compare between the up edge rate and the down edge rate, it then may be identify the character of the defect.

\section{Conclusion}

Practical application shows that this device and method have the characteristics of open, real-time, anti-jamming and high-performance. To apply this device in the nondestructive testing, will greatly increase system integration and reliability, improve the efficiency, reduce the development cycle and enhance the market competitiveness. Indepth study of the identify defect will have a certain practical significance to the development of non-destructive testing instruments.

\section{Acknowledgements}

This work was supported by National defense pre-research foundation 9140A27020113JB11393 and 9140A27020314JB11438.

\section{References}

\subsection{Journal Article}

[1] W. Luo, J. L. Rose and H. Kwun, "A two dimension model for crack in pipes", American Institute of Physics, vol. 5, no. 23, (2004).

[2] Y. Deng and W. Wang, "Boundary processing technique in EMD method and Hilbert transform, Chinese science Bulletin, vol. 11, no. 46, (2001).

[3] G. Yue, C. Kang and W. Miao, "The Aid Classification System for Metal Material Flaws in Ultrasonic Testing", Chinese Journal of Scientific Instrument, vol. 10, no. 26, (2005).

[4] B. Tang and H. Pan, "Circuit Board Signal Detection System Based on LabVIEW", Application of Electronic technology, vol. 2, no. 3, (2008).

[5] C.-J. Liao, X. J. Li and D. S. Liu, "Application of STFT in feature extraction of acoustic emission signal", Chinese Journal of Scientific Instrument, vol. 9, no. 29, (2008).

[6] D. X. Wu and X. L. Yang, "Identification of waveforms and defect in ultrasonic inspection", Nondestructive Testing, vol. 7, no. 24, (2012). 
[7] H. Kwun and S. Y. Kim, "Magnetostrictive Sensor for Generating and Detecting Plate Guided Waves", Journal of Pressure Vessel Technology.

[8] D. N. Alleyne, M. J. S. Lowe and C. Peter, "The Refection of guided waves From Circumferential Motches in Steel pipe", Journal of Applied Mechanics, vol. 5, no. 65, (1998).

[9] Z. X. Wu, "LabVIEW Emulation that the Signal Noise Elimination of Wavelet is designed", Journal of Nanping Teachers College, Journal of Nanping Teachers College, vol. 10, no. 24, (2005).

[10] G. J. Zang and Z. P. Liu, "Journal of East China Jiaotong University”, vol. 8, no. 24, (2007).

[11] J. R. Berriman and D. A. Hutchins, "“"The Application of Time-Frequency Analysis to the Air-Coupled Ultrasonic Testing of Concrete", IEEE Transactions on Ultrasonics, Ferroelectrics, and Frequency Control, vol. 4, no. 53, (2004).

[12] A. Demma, P. Cawley and M. Lowe, "The effect of Bends on the Propagation of guided Waves in Steel pipes", Pressure Vessel Technology, vol. 8, no. 127, (2005).

[13] M. J. Sablik, Y. C. Lu and G. L. Burkhardt, "Modeling magnetostrictive generation of elastic waves in steel pipes", International Journal of applied electro-magnetics and mechanics, vol. 8, no. 10, (1999).

\subsection{Book}

[14] X. Chen, "Design from entry to the master on LabVIEW8.20 Program", Tsinghua University Press (2007).

[15] A. Texas, "Joint Time-Frequency Analysis Toolkit Reference Manual", National Instruments Corporation, (1998).

\subsection{Conference Proceedings}

[16] S. He, H. Zhao and J. Zhou, "The optimal window length of STFT for sine modulated signal”, IET International Radar Conference, (2009); IEEE, Piscataway, USA.

[17] Chen, Ch. Tzu, Hung Cheng, "Time- frequency analysis in ultrasonic NDT.14 ${ }^{\text {th }}$ World Conference on NDT. (1996); India.

\section{Author}

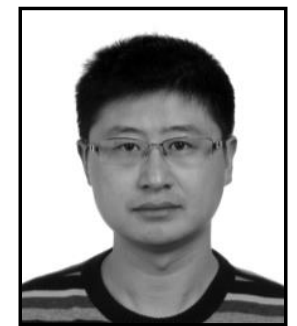

Yin Ming, Lecturer, he received the B.Eng. degree from the Electronic Engineering, Shandong University, Jinan, China, in 2002 and the M. Eng. degree from the System Engineering, Beijing Institute of Technology in 2008. He is currently working towards the Ph.D. degree at the school of Electronic Engineering, Naval University of Engineering, China. He has published more than 15 technical journal papers and international conference papers. His research interests include (1) condition monitoring, fault diagnosis, and aviation equipment maintenance; (2) and virtual instrument. 
International Journal of Signal Processing, Image Processing and Pattern Recognition Vol.8, No.6 (2015) 